\title{
Comparison of bladder autoaugmentation by transurethral vesicomyotomy and hydrodistention for ketamine cystitis
}

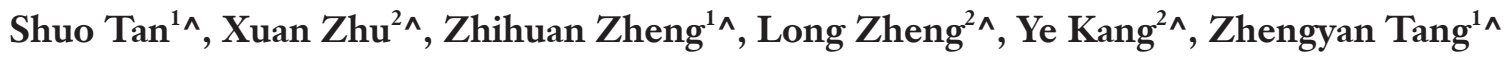 \\ ${ }^{1}$ Department of Urology, Xiangya Hospital, Central South University, Changsha, China; ${ }^{2}$ Department of Urology, The Second Xiangya Hospital, \\ Central South University, Changsha, China \\ Contributions: (I) Conception and design: Z Tang, S Tan; (II) Administrative support: Z Tang; (III) Provision of study materials or patients: X Zhu; \\ (IV) Collection and assembly of data: L Zheng, Z Zheng; (V) Data analysis and interpretation: Z Zheng, Y Kang, S Tan; (VI) Manuscript writing: All \\ authors; (VII) Final approval of manuscript: All authors. \\ Correspondence to: Prof. Zhengyan Tang. Department of Urology, Xiangya Hospital, Central South University, 87 Xiangya St., Changsha 410008, \\ China. Email: xytzyan@163.com.
}

\begin{abstract}
Background: To illustrate the bladder autoaugmentation by transurethral vesicomyotomy (BATV) and compare the efficacy and safety of BATV to bladder hydrodistention (BH) for managing ketamine cystitis (KC). Methods: We retrospectively analyzed clinical data for 53 patients with $\mathrm{KC}$ who received surgical intervention between 2014 and 2019 at our hospital. Of these, 41 (77.4\%) underwent BH and 12 (22.6\%) were subjected to BATV, with a minimum of 1-year follow-up. These groups were compared with reference to urodynamic parameters, subjective symptom scores as well as all complications.

Results: Both groups were matched in age, addiction time, preoperative urodynamic parameters, postvoid residual urinary volume (PVR), and symptom scores. All urodynamic parameters including maximum cystometric capacity (MCC), maximum detrusor pressure $\left(\mathrm{P}_{\mathrm{det} \max }\right)$, compliance, maximum urinary flow rate $\left(\mathrm{Q}_{\max }\right)$ and symptom scores had improved significantly in two groups whether at 3 or 12 months. Moreover, the MCC was significantly increased after BATV than BH, at a mean [standard deviation (SD)] of 281.0 (25.7) vs. 213.5 (35.6) $\mathrm{mL}(\mathrm{P}<0.001)$ at 12 -month follow-up. The $\mathrm{Q}_{\max }$ and the Pelvic Pain and Urgency/Frequency (PUF) symptom score were still noted better in the BATV group at 3 months after surgery. Additionally, patients in both groups had similarly low rates of complications.
\end{abstract}

Conclusions: BATV is superior to BH with increased bladder capacity and urodynamic outcomes, although showing equivalent in symptom relief and a similar rate of complications.

Keywords: Ketamine; cystitis; bladder autoaugmentation; vesicomyotomy; hydrodistention

Submitted Mar 04, 2021. Accepted for publication Apr 16, 2021.

doi: $10.21037 /$ tau-21-188

View this article at: http://dx.doi.org/10.21037/tau-21-188

\section{Introduction}

Ketamine, an antagonist of the N-methyl-D-aspartic acid (NMDA) receptor complex, is largely used for anesthesia and as a therapy for mental disorders (1). With increasing ketamine abuse in adolescents as a recreational drug, ketamine cystitis (KC), one of its adverse health effects, has become a new clinical problem affecting the genitourinary tract. Pelvic pain, hematuria and lower urinary tract symptoms (LUTS) characterized by dysuria and urinary frequency/urgency have occurred globally in $26.6 \%$

\footnotetext{
^ ORCID: Shuo Tan, 0000-0003-2655-5314; Xuan Zhu, 0000-0002-4404-4393; Zhihuan Zheng, 0000-0003-1610-5125; Long Zheng, 0000-0001-9126-7058; Ye Kang, 0000-0001-6630-7898; Zhengyan Tang, 0000-0003-0693-8748.
} 
of ketamine abusers (2). In addition, some severe cases progressed to bladder contracture and vesicoureteral reflux, leading to hydronephrosis of the upper urinary tract and ultimately impaired renal function (3).

Conservative treatments including behavior therapy and medication have provided little relief despite the cessation of ketamine $(4,5)$. Surgical interventions including hydrodistention, augmentation cystoplasty, autoaugmentation, ureteric re-implantation, and cystectomy with neobladders have been reported sporadically for patients with intractable and relapse KC (6-8). Bladder hydrodistention was first suggested for interstitial cystitis/ bladder pain syndrome (IC/BPS) in 1922 and also reported short-term improved bladder capacity as well as relieved LUTS for KC in spite of the limited experience $(8,9)$. As treatment efficacy of hydrodistention decreased with longterm follow-up, more aggressive surgeries like bladder augmentation by cystoplasty have been performed and shown sustainably effective for $\mathrm{KC}$, but is also associated with a high risk of complications and failure rates $(6,7,10,11)$. Thus, BATV, which was initially used for contracted bladder, strives to offer a novel and stable therapeutic response to $\mathrm{KC}$ as minimally invasive alternatives along with a lower risk of adverse events (12). Here, we present 1-year retrospective results comparing BATV and BH as treatments for $\mathrm{KC}$ with regard to symptom relief and improvements in urodynamic outcomes. We present the following article in accordance with the STROBE reporting checklist (available at http://dx.doi.org/10.21037/tau-21-188).

\section{Methods}

\section{Participants}

The study was conducted in accordance with the Declaration of Helsinki (as revised in 2013). The study was approved by the Ethics Committee of Xiangya Hospital, Central South University (NO.: 202008101) and individual consent for this retrospective analysis was waived. Between 2014 and 2019, we retrospectively reviewed 61 patients with $\mathrm{KC}$ who underwent surgical intervention in our institution. All of these patients were unresponsive to conservative treatment preoperatively including anticholinergic medication, pelvic floor therapy and/or intravesical treatments. Indications for surgery included upper tract deterioration, maximum cystometric capacity (MCC) $<150 \mathrm{~mL}$, bladder compliance $<30 \mathrm{~mL} / \mathrm{cmH}_{2} \mathrm{O}$, refractory and/or relapse KC. No patients treated firstly by $\mathrm{BH}$ then BATV were enrolled in the study. Besides, 4 of patients with difficulty in transurethral operation and 4 with missing data were excluded. Overall, the study identified 53 patients, of whom 41 (77.4\%) underwent BH and $12(22.6 \%)$ were treated by BATV. There has been no standardized diagnose of KC. All patients provided a detailed history of ketamine use and underwent physical examination as well as urodynamics testing. Preoperative cystourethroscopy and biopsy are performed on initial assessment of KC. All excluded other pathology such as IC/BPS, neurogenic bladder or tuberculosis affecting the bladder and confirmed the typical pathological changes containing denuded mucosa, inflammation with lymphocyte and monocyte infiltration, and fibrosis of the bladder wall (6). Additionally, KC associated LUTS were quantitatively evaluated by Pelvic Pain and Urgency/Frequency (PUF) symptom score, Visual Analogue Scale/Score (VAS), O'Leary-Sant IC Symptom Index (ICSI), IC Problem Index (ICPI), and Quality of Life (QoL) score. The urinary tract was determined by computerized tomography (CT) scans while postvoid residual urinary volume (PVR) by immediate transurethral bladder drainage, and urodynamic testing was applied to assess MCC, maximum detrusor pressure $\left(\mathrm{P}_{\text {det max }}\right)$, compliance and maximum urinary flow rate $\left(\mathrm{Q}_{\max }\right)$. Peri- and postoperative outcomes measured in two groups included operative time, duration of catheterization, hospital stay and complications. The catheter was subsequently removed after the urine turned reddish or clear, and patients were discharged 48 hours after removal of the catheter. All patients were evaluated for bladder capacity, LUTS and adverse events along with imaging and urodynamic assessment at the 3-and 12-month follow-ups.

\section{Interventions}

A single experienced surgeon at our site conducted all procedures using general anesthesia in the operating suite. BATV was performed using an Olympus cystoresectoscope (Japan, A22001), and patients were placed in a lithotomy position. During the operation, the surgeon examined the walls, neck and trigone of bladder and marked bilateral ureteric orifices aiming to avoid iatrogenic injury. Next, the mucosa and detrusor of bladder were incised longitudinally and latitudinally under resectoscope to create the diverticulum until perivesical fat was vaguely visible. The depth of incision varies from 1 to $2.5 \mathrm{~mm}$ due to the thickness of the bladder wall. Urethral catheter drainage 
was instituted and then the catheter was removed until postoperative hematuria receded.

Since there have been no standard methods of hydrodistention, we suggest performing low-pressure and short-duration BH similar to that described by Hanno (13). After general anesthesia was administered in each case, an initial cystoscopic examination was performed and urine obtained for cytology. The tube was subsequently inserted into the bladder meanwhile its tail connected a pressure monitor. A saline serum pouch was then suspended 60 to 80 centimeters above the operating table. The perfusion procedure began and continued to ensure the pressure was maintained approximately at $60 \mathrm{cmH}_{2} \mathrm{O}$ for 10 minutes without rest intervals. While inactive bleeding was recorded in a re-examined cystoscopy, the bladder was later continuously rinsed through the indwelling F22 3-way silicone catheter.

\section{Statistical analysis}

Pearson's Chi-squared and Fisher's exact tests were used for categorical characteristics as appropriate. Mann-Whitney $\mathrm{U}$ test was used for continuous variables as a comparison between baseline and 3-12 months. A value of $\mathrm{P}<0.05$ was considered statistically significant(two-tailed). Data analysis was conducted using SPSS, version 22.0 (SPSS Inc., IBM Corp, USA).

\section{Results}

\section{Baseline}

The mean age $(25.0 \pm 5.1$ vs. $25.2 \pm 3.8$ years $)$, addiction time (3.0 0.9 vs. $3.3 \pm 1.7$ years) and gender $(75.0 \%$ vs. $73.2 \%$ of men) were not different between the BATV and BH groups (Table 1). The mean [standard deviation (SD)] preoperative MCC was 92.2 (12.3) mL before BATV surgery, compared to $93.2(19.1) \mathrm{mL}$ in the $\mathrm{BH}$ group. Besides, other urodynamic parameters, PVR and LUTS scores were similar between the two groups. Cystoscopy and histology show denuded mucosa, submucosal edema, microvascular damage, inflammation with lymphocyte and monocyte infiltration, and fibrosis of the bladder wall.

\section{Operative characteristics}

With reference to the perioperative data, operative time in the BATV group was significantly longer than BH
$(90.3 \pm 12.5$ vs. $52.7 \pm 12.4 \mathrm{~min}, \mathrm{P}<0.001)$. The estimated blood loss was not high enough to be evaluated in both groups because of the minimal invasion. In addition, the hospital stay and catheterization time showed similarities between the two groups (Table 1).

\section{Treatment efficacy}

As shown in Tables 2,3, all functional outcomes including MCC, $\mathrm{Q}_{\max }$, and symptom scores were significantly improved in both groups compared to baseline at 3and 12-month follow-up. In comparison with 3-month, the mean MCC and $\mathrm{Q}_{\max }$ were not further increased at 12 -month in both groups. Also, the symptom scores at 12-month follow-up were not noted a remarkable reduction than at 3-month. Nevertheless, a significant increase in PVR was observed at 12 -month follow-up than baseline after BATV $(46.3 \pm 3.6$ vs. $15.4 \pm 5.2 \mathrm{~mL})$.

The MCC in the BATV group was notably higher than in the $\mathrm{BH}$ group $(281.0 \pm 25.7$ vs. $213.5 \pm 35.6 \mathrm{~mL}, \mathrm{P}<0.001)$ at 12-month follow-up. The BATV still provided better improvements in compliance and $\mathrm{P}_{\operatorname{det} \max }$. Furthermore, the BATV group achieved a better outcome in PUF scores and $\mathrm{Q}_{\max }(\mathrm{P}=0.005, \mathrm{P}=0.021$ respectively) at 3-month follow-up while showed insignificant difference at 12-month compared to the BH group. But the BATV group was demonstrated a more increased PVR at 12-month after surgery (46.3 \pm 3.6 vs. $15.9 \pm 4.7 \mathrm{~mL}$ ) (Table 2, Figure 1). Regarding any other follow-up parameters to evaluate the efficacy of both approaches, there were no significant differences between two groups within 12 months of follow-up.

Patients with preoperative $\mathrm{MCC}<100 \mathrm{~mL}$ show more significant improvement in MCC, compliance and $\mathrm{P}_{\text {det max }}$ after BATV than MCC $\geq 150 \mathrm{~mL}$ (Figure 2). Cumulative percentages of patients achieving various improvements in bladder capacity and $\mathrm{Q}_{\max }$ in both groups are expressed in Figure 3. The increase in bladder capacity after BATV shows better than $\mathrm{BH}$ at 12 months from baseline $(189.1 \pm 16.8$ vs. $119.6 \pm 31.7 \mathrm{~mL}, \mathrm{P}<0.001)$ while the changed volumes of PVR in two groups differ significantly $(30.7 \pm 4.0$ vs. $-0.3 \pm 6.4 \mathrm{~mL}, \mathrm{P}<0.001$ ) (Table 2, Figure 2). Beyond that, the changes in $\mathrm{Q}_{\max }$ and subjective symptom scores had no significant differences.

\section{Complications}

Thirteen patients $(26.5 \%)$ of all encountered complications 
Table 1 Baseline characteristics and perioperative outcomes of the included patients

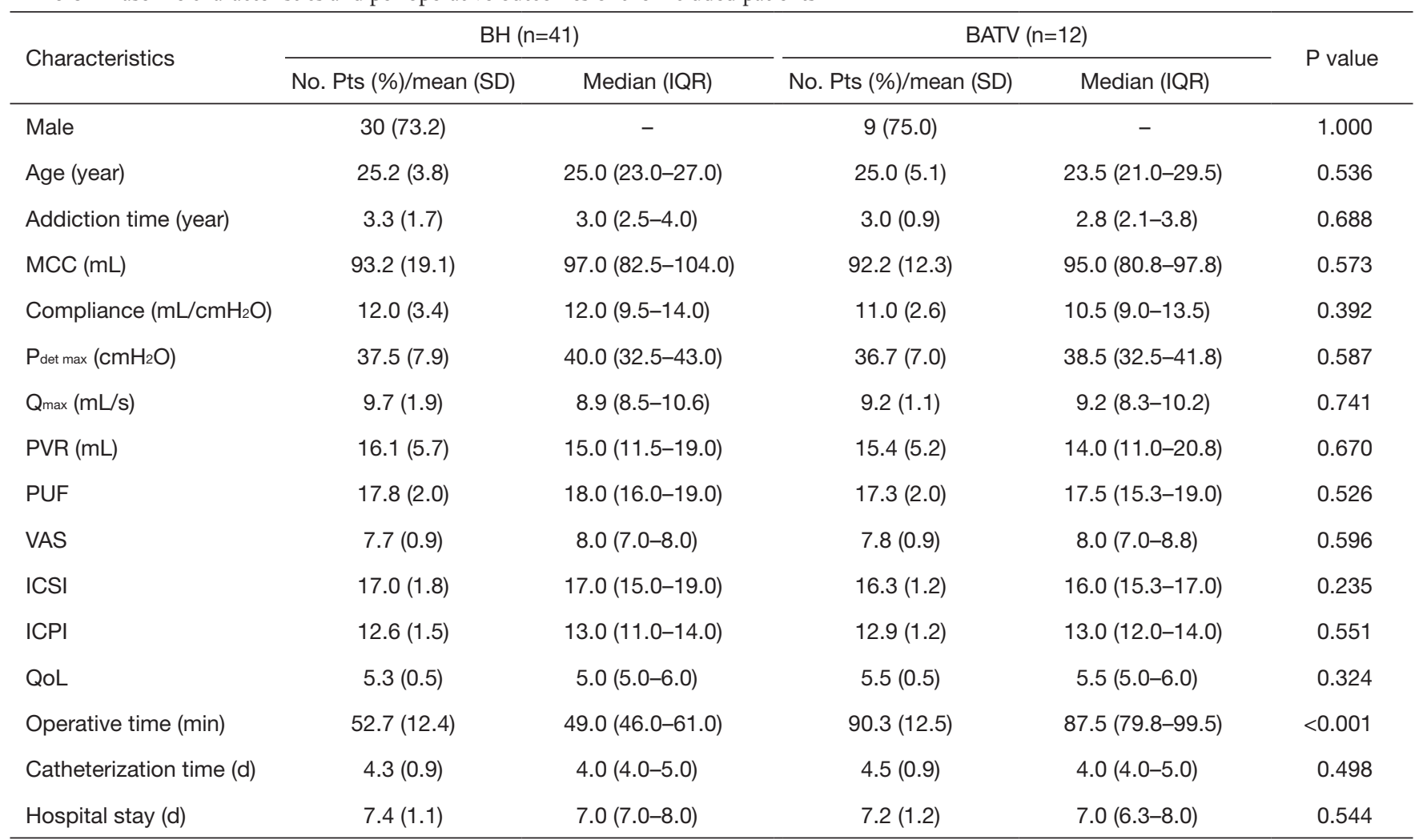

$\mathrm{BH}$, bladder hydrodistention; BATV, bladder autoaugmentation by transurethral vesicomyotomy; Qmax, maximum urinary flow rate; MCC, maximum cystometric capacity; Pdet max, maximum detrusor pressure; PVR, postvoid residual urinary volume; PUF, the Pelvic Pain and Urgency/Frequency; VAS, Visual Analogue Scale/Score; ICSI, O'Leary-Sant IC Symptom Index; ICPI, IC Problem Index; QoL, quality of life.

except 4 patients lost to follow-up within 12 months. Complications were classified using the Clavien-Dindo scale (grades I-IV) and most of these were Clavien grade I and II (Table 4). In the BH group, recurrent or persistent LUTS was the predominant complication while the rate of which was not significantly higher than in the BATV group. Moderate complications including bladder perforation and stone formation were present respectively in one patient and another after BATV (9.1\%, P=0.224). Correspondingly, prolonged catheterization and transurethral cystolitholapaxy and lithotripsy were offered. Chronic urinary retention, acute kidney injury, and venous thromboembolism (VTE) were noted in 1 patient from each group, and these cases were treated with subsequent treatment including clean intermittent catheterization, diuretics along with hemodynamic management, and anticoagulant respectively. In addition, $3(7.9 \%)$ patients in the $\mathrm{BH}$ group reported urinary tract infections (UTIs) than $1(9.1 \%)$ in the BATV group, and one patient after $\mathrm{BH}$ developed sepsis requiring culture-based antimicrobial. Four (10.5\%) and 2 (18.2\%) of patients who underwent BH and BATV respectively had hypogastric pain and demanded analgesics. No patient after BATV and $4(10.5 \%)$ patients after $\mathrm{BH}$ received continuous bladder irrigation due to gross hematuria. Overall, there was no significant difference in the incidence of complications in two groups.

\section{Discussion}

According to recent studies, cessation of ketamine is the mainstay of relieving LUTS, improving bladder capacity and avoiding further deterioration of renal function in spite of its difficulty for frequent users $(14,15)$. Previous reports have suggested unsatisfactory results of conservative managements and that surgical interventions have been considered suitable for these patients who had contracted bladders, refractory and recurrent symptoms or upper tract compromise $(6,7,15)$. Sihra et al. (7) reviewed ketamine abusers treated with urinary tract reconstruction such as augmentation cystoplasty, total cystectomy with orthotopic 
Table 2 Functional outcomes at 3 and 12 months of follow-up

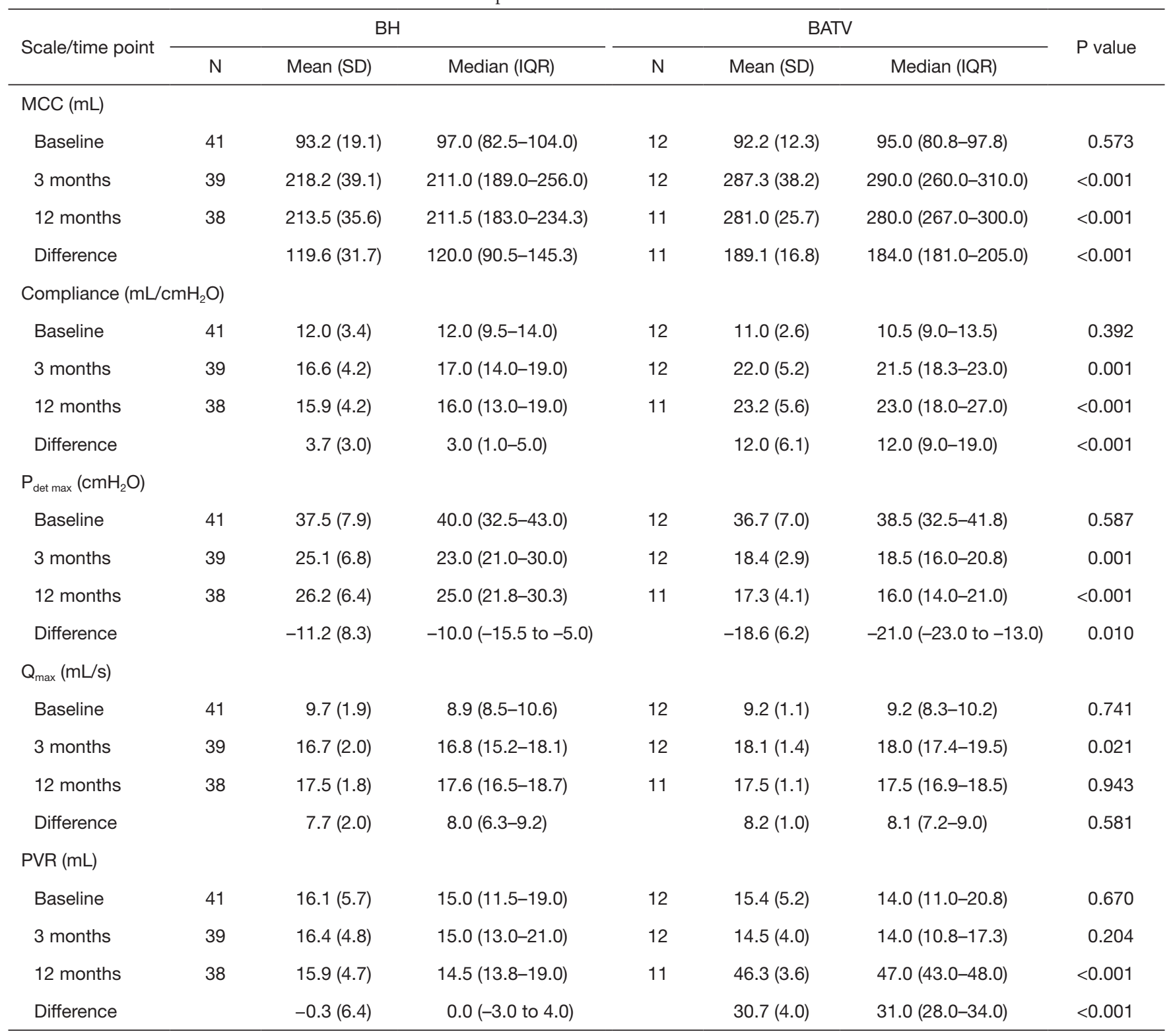

$\mathrm{BH}$, bladder hydrodistention; BATV, bladder autoaugmentation by transurethral vesicomyotomy; Qmax, maximum urinary flow rate; MCC, maximum cystometric capacity; Pdet max, maximum detrusor pressure; PVR, postvoid residual urinary volume.

neobladder formation and heterotopic neobladder with Mitrofanoff, and deemed that they developed significant higher rate of postoperative complications than patients who underwent these surgeries for other benign diseases like refractory IC/BPS (71.4\% vs. $37 \%)$. Some severe complications requiring surgical interventions remarkably impaired QoL of patients with KC who were afterward deprived of further managements and thus must be carefully selected and appropriately counseled for reconstructive surgery.

In the last decade, BH, BATV and other alternative surgery such as bladder autoaugmentation by inflatable silicone balloon and autoaugmentation by vesicomyotomy have emerged as minimally invasive approaches for patients with contracted bladder or intractable LUTS caused by KC, IC/BPS or neuropathic bladders after failed conservative therapy $(8,12,16,17)$. Both Yang et al. (8) and Zeng et al. (9) have suggested their clinical outcomes of 18 and 36 patients 
Table 3 Comparison of subjective symptom questionnaires

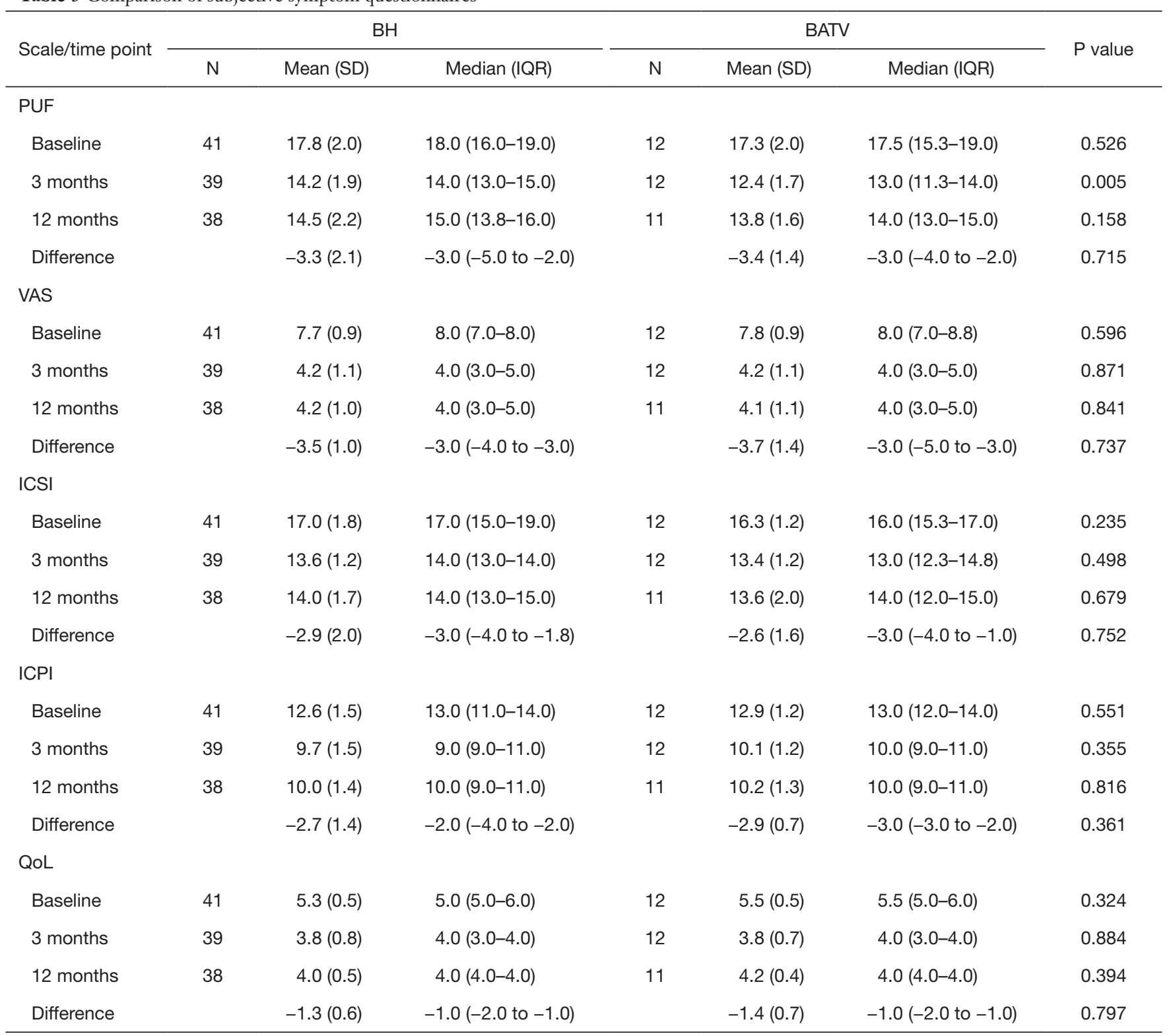

$\mathrm{BH}$, bladder hydrodistention; BATV, bladder autoaugmentation by transurethral vesicomyotomy; PUF, the Pelvic Pain and Urgency/ Frequency; VAS, Visual Analogue Scale/Score; ICSI, O'Leary-Sant IC Symptom Index; ICPI, IC Problem Index; QoL, quality of life.

with $\mathrm{KC}$ who underwent $\mathrm{BH}$ respectively and confirmed increased bladder capacity and subjective symptom relief in short-term follow-up. Nevertheless, there is still some study (18) shows their failure to achieve efficacy of BH for treating $\mathrm{KC}$ and presumably attributed it to inadequate damage to detrusor and mucosal afferent nerve endings, which followed the similar principle previously used in IC/ BPS $(8,19)$.

Autoaugmentation was firstly described as a method to improve bladder compliance and capacity by Cartwright and Snow $(20,21)$ and modified by numbers of surgeons, each of whom named the procedure differently depending on whether the detrusor muscle was incised or excised. Stothers et al. (22) concluded that autoaugmentation by vesicomyotomy offered no advantage over vesicomyotomy in 12 patients with neurogenic bladder. Also, they revealed a mean $40 \%$ increase in bladder capacity as well as minor complications in patients who underwent vesicomyotomy. 
A

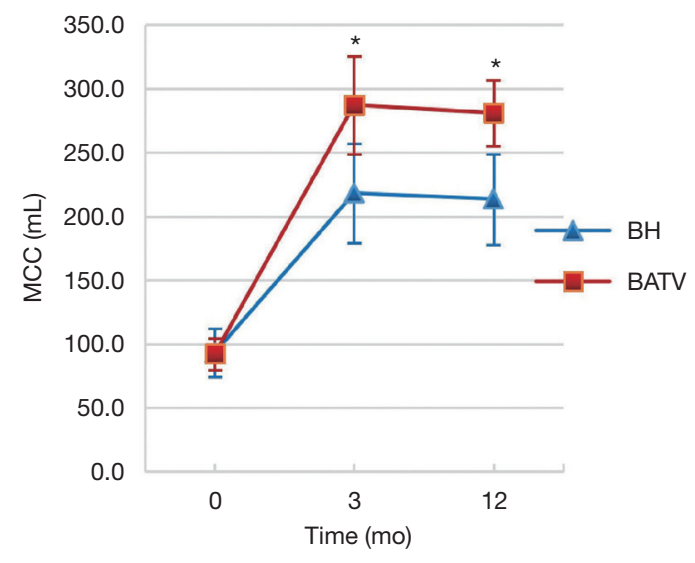

C

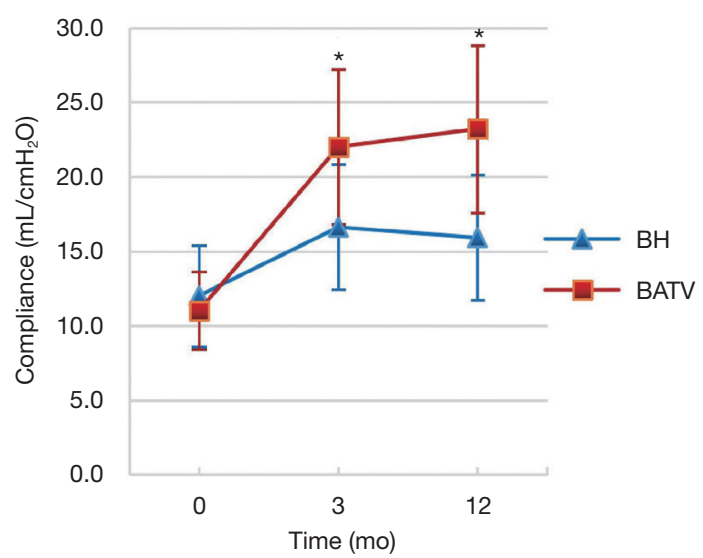

B

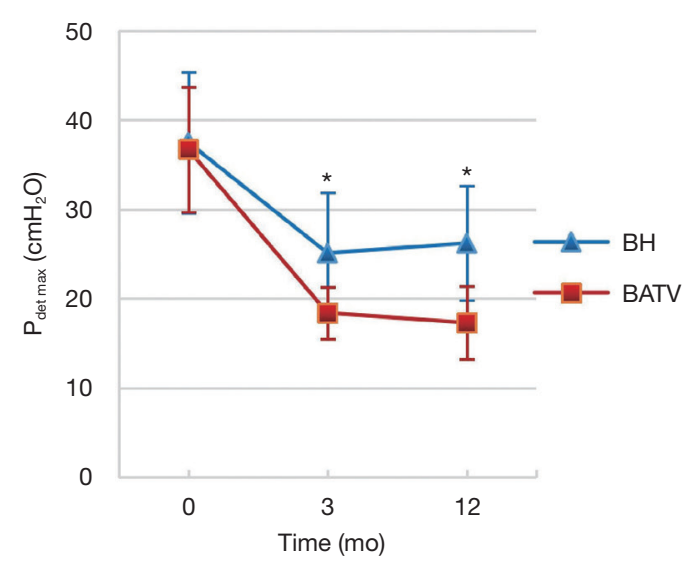

D

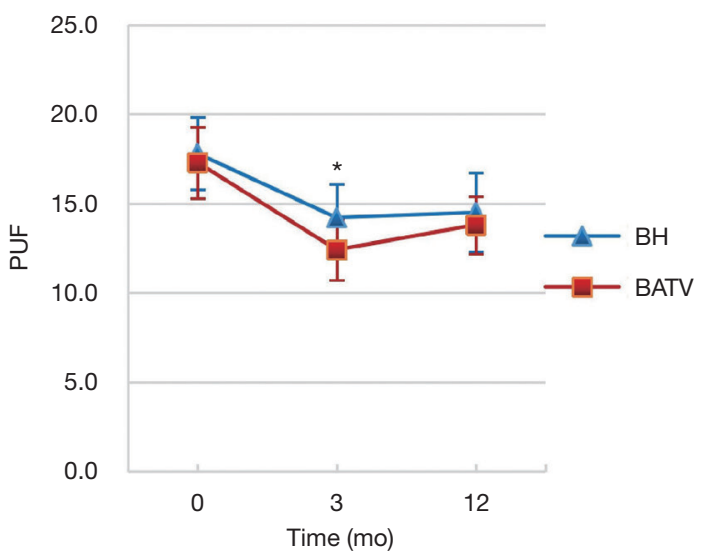

Figure 1 Outcomes following intervention with BH and BATV were assessed in terms of maximum cystometric capacity (A), maximum detrusor pressure $\left(\mathrm{P}_{\text {det max }}\right)(\mathrm{B})$, compliance $(\mathrm{C})$, the Pelvic Pain and Urgency/Frequency (PUF) (D) within 12 months. The mean with $95 \%$ confidence interval of each parameter is indicated. * shows significant difference in two groups. BH, bladder hydrodistention; BATV, bladder autoaugmentation by transurethral vesicomyotomy.

Akin to treatment strategy to small and poorly compliant neurogenic bladders, BATV was performed as an intervention to $\mathrm{KC}$ in our series which showed similar short-term improvement in urodynamic parameters. This study is important and groundbreaking since systematic assessment of efficacy and safety of both BATV and BH procedure applied to $\mathrm{KC}$ is still rarely reported.

The present study shows urodynamic improvement along with decreases in symptom scores including PUF, ICSI, ICPI, VAS, QoL in both groups at 3 -month and which were maintained for the duration of 1-year followup. In addition, the comparison appears to slightly favor patients treated with BATV when analyzing urodynamic outcomes. But as clearly shown, PVR was significantly increased after BATV at the 12-month follow-up while there was no significant change in $\mathrm{BH}$ group at any followup interval. The acquired bladder diverticula with short diameter were found initially after BATV due to the incision of detrusor muscle and the incisional fibrosis of the bladder wall. And the advantage of post-operative $\mathrm{Q}_{\max }$ at 3-month follow-up after BATV disappeared at 12-month along with significantly increased PVR volume, which was presumably attributed to the expansion of bladder diverticulum and the fibrotic contracture of bladder inner wall. However, this was still acceptable since the mean (SD) PVR volume in BATV group at 12-month follow-up was 46.3 (3.6) mL 

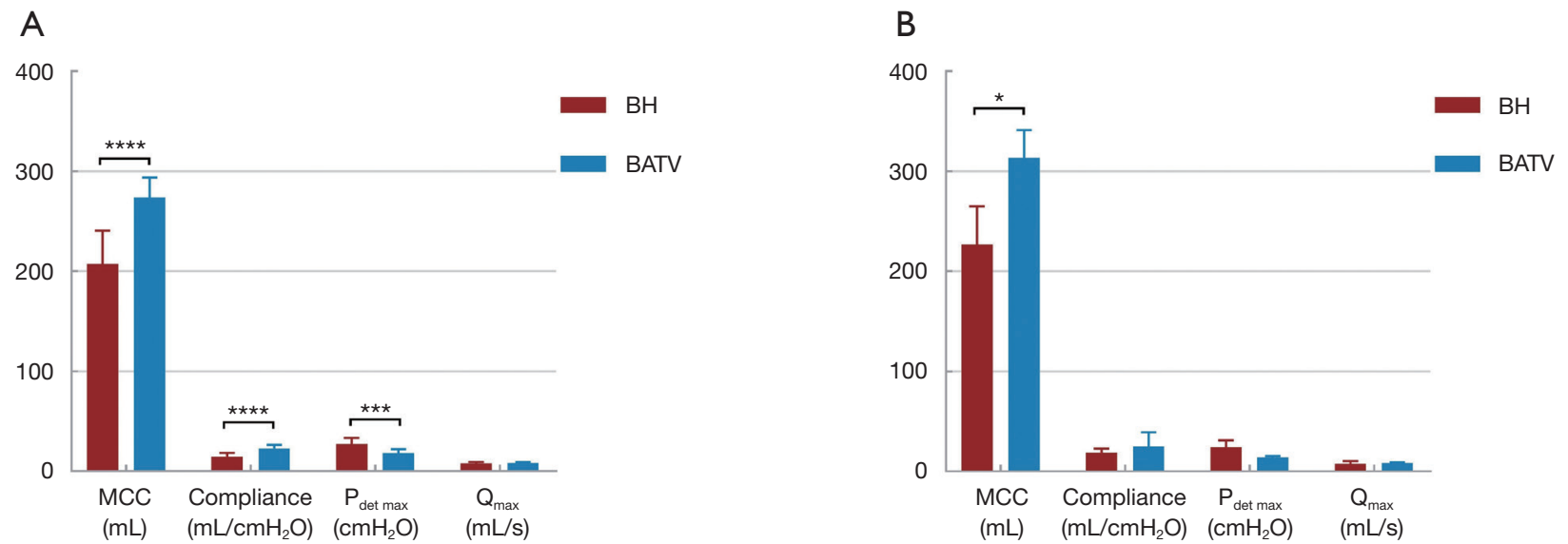

Figure 2 Comparison of urodynamic parameters at 12 -month follow-up in patients with preoperative MCC $<100 \mathrm{~mL}$ (A), and patients with preoperative $\mathrm{MCC} \geq 100 \mathrm{~mL}(\mathrm{~B})$. Compared with the $\mathrm{BH}$ group, ${ }^{*}, \mathrm{P}<0.05 ;{ }^{* * *}, \mathrm{P}<0.001$; ${ }^{* * *}, \mathrm{P}<0.0001$. BH, bladder hydrodistention; BATV, bladder autoaugmentation by transurethral vesicomyotomy; $\mathrm{Q}_{\max }$, maximum urinary flow rate; MCC, maximum cystometric capacity; $\mathrm{P}_{\text {det max }}$, maximum detrusor pressure.

A

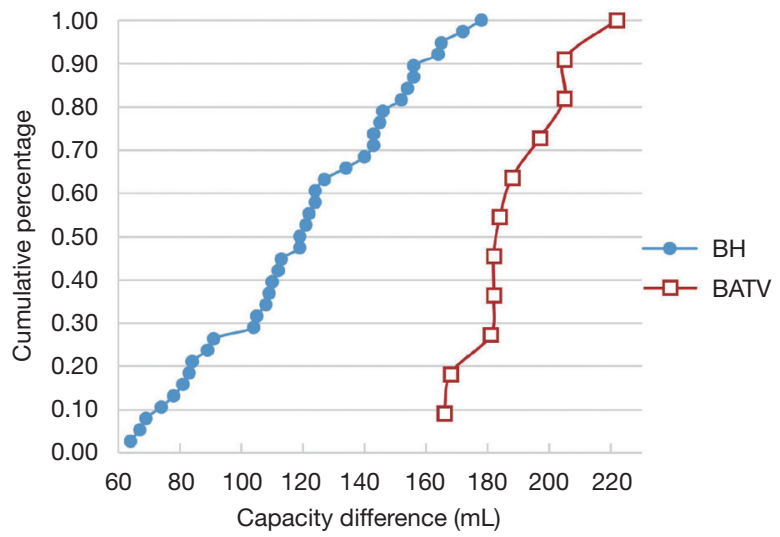

B

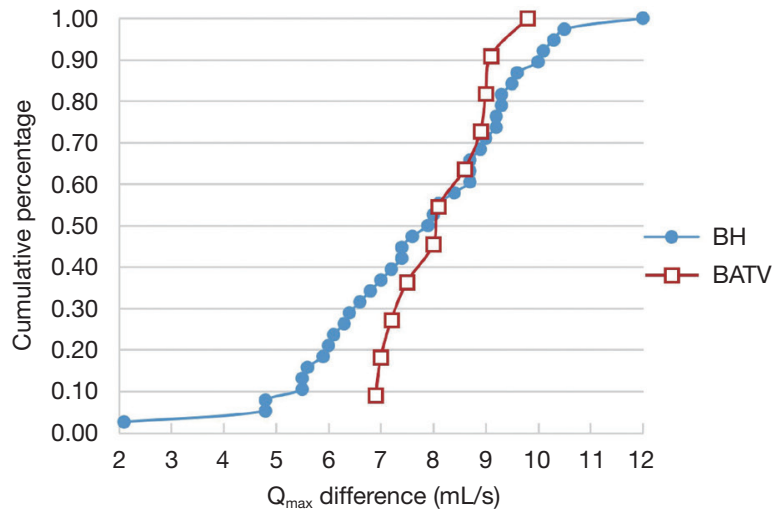

Figure 3 Cumulative percentages of changes in (A) maximum cystometric capacity and (B) maximum urinary flow rate (12 months after surgery vs. baseline) in the $\mathrm{BH}$ and BATV groups, e.g., half the patients received an increase in bladder capacity $\geq 120 \mathrm{~mL}$ after $\mathrm{BH}$ compared to $180 \mathrm{~mL}$ after BATV. BH, bladder hydrodistention; BATV, bladder autoaugmentation by transurethral vesicomyotomy.

and also lower than reported volume of other alternative surgery for $\mathrm{KC}$ [for instance, $82.6 \mathrm{~mL}$ of augmentation enterocystoplasty reported by Jhang et al. (15)]. Besides, in our series, where patients with slightly increased PVR, renal function had still not been implied in long-term follow-up the same as the surgical intervention for KC reported by Sihra et al. (7).

In a recent study of 81 patients who underwent hydrodistention and augmentation for treating $\mathrm{KC}, \mathrm{Wu}$ et al. (23) found a significant lower PUF score in augmentation group $(11.5 \pm 1.6$ vs. $17.2 \pm 3.6)$. Similarly, we found that BATV resulted in better PUF score than BH at 3 months in the present study $(12.4 \pm 1.7$ vs. $14.2 \pm 1.9$, $\mathrm{P}=0.005)$. This may be related to severer vesicomyotomyinduced lesions to afferent nerve endings than other approaches. Postoperative cystoscopy showed that the bladder volume was significantly improved and the bladder filling time was prolonged at same low flow and pressure than preoperative examination. Furthermore, the hypervascular reddened mucosal areas were reduced as well 
Table 4 Treatment-related complications after BATV and BH stratified by Clavien-Dindo classification grade

\begin{tabular}{|c|c|c|c|c|}
\hline Complications & No. BH (\%) & No. BATV (\%) & $P$ value & Interventions \\
\hline \multicolumn{5}{|l|}{ Clavien-Dindo grade I } \\
\hline Chronic urinary retention & $1(2.6)$ & $1(9.1)$ & 0.402 & Clean intermittent catheterization \\
\hline Acute kidney injury & $1(2.6)$ & $1(9.1)$ & 0.402 & Diuretics/haemodynamic management \\
\hline Gross hematuria & $4(10.5)$ & 0 & 0.562 & Continuous bladder irrigation \\
\hline Recurrent/persistent LUTS & $7(18.4)$ & $2(18.2)$ & 1.000 & Anticholinergic medications \\
\hline \multicolumn{5}{|l|}{ Clavien-Dindo grade II } \\
\hline Venous thromboembolism & $1(2.6)$ & $1(9.1)$ & 0.402 & Anticoagulant \\
\hline Urinary tract infections & $3(7.9)$ & $1(9.1)$ & 1.000 & Culture based antimicrobial \\
\hline \multicolumn{5}{|l|}{ Clavien-Dindo grade IIla } \\
\hline Bladder perforation & 0 & $1(9.1)$ & 0.224 & Prolonged catheterization \\
\hline \multicolumn{5}{|l|}{ Clavien-Dindo grade IIIb } \\
\hline Bladder stone formation & 0 & $1(9.1)$ & 0.224 & Transurethral cystolitholapaxy and lithotripsy \\
\hline Total & $10(26.3)$ & $3(27.3)$ & 0.614 & \\
\hline $\begin{array}{l}\text { Complications in patients with } \\
\text { preoperative } \mathrm{MCC}<100 \mathrm{~mL}\end{array}$ & $6(23.1)$ & $3(33.3)$ & 0.421 & - \\
\hline $\begin{array}{l}\text { Complications in patients with } \\
\text { preoperative } \mathrm{MCC} \geq 100 \mathrm{~mL}\end{array}$ & $4(33.3)$ & 0 & 0.495 & - \\
\hline
\end{tabular}

$\mathrm{BH}$, bladder hydrodistention; BATV, bladder autoaugmentation by transurethral vesicomyotomy; LUTS, lower urinary tract symptoms; MCC, maximum cystometric capacity.

as the fibrous scar after both surgeries. But the cystoscopic superiority has not been found in the remission of chronic inflammation of the bladder wall after BATV than the BH group. Comparing the subjective symptom scores after interventions in two groups, there were no significant differences at long-term follow-up as well. Persistent panurothelial or neurogenic inflammation with ischemia due to preoperative high and prolonged ketamine exposure may be some reason for this similarity despite the improved MCC and the incised overactive detrusor between the two groups.

Regarding postoperative complications, the overall rate of which was not significantly different between BATV group $(27.3 \%)$ and $\mathrm{BH}$ group (25.6\%), and was consistent with previous reports $(8,12,17,20,22-24)$. The total complication rate in both groups thus strikingly lower than other bladder augmentation approaches such as cystoplasty, the rate of which ranged between $71 \%$ and $90 \%(7,10,11)$. Moreover, both procedures were minimally invasive with low morbidity in Clavien grade III + complications as well as relatively uncomplicated interventions. It was speculated that the reason may be the absence of invasive urinary tract reconstructive procedure along with higher grade adverse events such as anastomotic leaks, strictures or bowel obstruction. And the patients with preoperative MCC $<100 \mathrm{~mL}$ demonstrated non-inferiority in safety after BATV while showed better urodynamics to the $\mathrm{BH}$ groups. Small bladder with thick fibrous wall may benefit more from BATV than $\mathrm{BH}$, possibly because of the inadequate expansion of bladder under the pressure of $60 \mathrm{cmH}_{2} \mathrm{O}$ during hydrodistention.

BATV seems slightly superior to $\mathrm{BH}$ in terms of preventing post-operative gross hematuria although the difference between two groups was not statistically significant. The BATV electrode enables the surgeon to coagulate any bleeding when detrusor muscle was incised and thus provided excellent visibility during the operation. Additionally, the rate of UTIs appears analogue (7.9\% vs. $9.1 \%)$ together with similar duration of catheterization 
(4.3 vs. 4.0 days) between two groups. And one patient after $\mathrm{BH}$ developed sepsis and subsequently treated with culture-based antimicrobial. However, unlike catheterinduced UTIs, some patients with pre-operative bacteriuria caused by prolonged urinary dysfunction may be the main pathogenesis of UTIs after surgery. Similar to our experience, both Chu et al. (3) and Yang et al. (8) revealed that almost all patients suffered from KC combined with pre-operative bacteriuria and elevated urinary leukocytes. Therefore, adequate preoperative urinalysis and prophylactic use of culture-based antibiotics are necessary to prevent postoperative UTIs for KC after surgery.

There are several limitations in the present study and the main one exists in its retrospective design. Moreover, the insufficient sample size enrolled in this study combined with short-term follow-up weakens the significance of data. A more accurate elucidation to the efficacy and safety of BATV and BH warrants larger pool of cases, prospective and randomized controlled design as well as long-term follow-up. Nevertheless, this study provides satisfactory peri-operative and 12-month follow-up data and systematically analyzes the differences in outcomes between BATV and BH procedures in spite of above drawbacks. Therefore, the findings will be helpful in selecting appropriate surgical approaches for $\mathrm{KC}$.

\section{Conclusions}

BATV is a safe and efficacious procedure as a minimallyinvasive alternative in treating KC. Besides, 1-year followup data shows BATV is superior to $\mathrm{BH}$ in terms of increasing urodynamic outcomes, and has similar rate of complications as well as symptom relief. Whilst it may not have broad indications in treatment of $\mathrm{KC}$, it provides an option for patients situated in the cure pathway between conservative therapy and urinary tract reconstruction.

\section{Acknowledgments}

Funding: None.

\section{Footnote}

Reporting Checklist: The authors have completed the STROBE reporting checklist. Available at http://dx.doi. org/10.21037/tau-21-188
Data Sharing Statement: Available at http://dx.doi. org/10.21037/tau-21-188

Peer Review File: Available at http://dx.doi.org/10.21037/ tau-21-188

Conflicts of Interest: All authors have completed the ICMJE uniform disclosure form (available at http://dx.doi. org/10.21037/tau-21-188). The authors have no conflicts of interest to declare.

Ethical Statement: The authors are accountable for all aspects of the work in ensuring that questions related to the accuracy or integrity of any part of the work are appropriately investigated and resolved. The study was conducted in accordance with the Declaration of Helsinki (as revised in 2013). The study was approved by the Ethics Committee of Xiangya Hospital, Central South University (NO.: 202008101) and individual consent for this retrospective analysis was waived.

Open Access Statement: This is an Open Access article distributed in accordance with the Creative Commons Attribution-NonCommercial-NoDerivs 4.0 International License (CC BY-NC-ND 4.0), which permits the noncommercial replication and distribution of the article with the strict proviso that no changes or edits are made and the original work is properly cited (including links to both the formal publication through the relevant DOI and the license). See: https://creativecommons.org/licenses/by-nc-nd/4.0/.

\section{References}

1. Yang Y, Cui Y, Sang K, et al. Ketamine blocks bursting in the lateral habenula to rapidly relieve depression. Nature 2018;554:317-22.

2. Winstock AR, Mitcheson L, Gillatt DA, et al. The prevalence and natural history of urinary symptoms among recreational ketamine users. BJU Int 2012;110:1762-6.

3. Chu PS, Ma WK, Wong SC, et al. The destruction of the lower urinary tract by ketamine abuse: a new syndrome? BJU Int 2008;102:1616-22.

4. Middela S, Pearce I. Ketamine-induced vesicopathy: a literature review. Int J Clin Pract 2011;65:27-30.

5. Shahani R, Streutker C, Dickson B, et al. Ketamineassociated ulcerative cystitis: a new clinical entity. Urology 2007;69:810-2. 
6. Chung SD, Wang CC, Kuo HC. Augmentation enterocystoplasty is effective in relieving refractory ketamine-related bladder pain. Neurourol Urodyn 2014;33:1207-11.

7. Sihra N, Ockrim J, Wood D. The effects of recreational ketamine cystitis on urinary tract reconstruction - a surgical challenge. BJU Int 2018;121:458-65.

8. Yang XS, Chen Z, Duan JL, et al. Efficacy of cystectasia in the treatment of ketamine-induced bladder contracture. Transl Androl Urol 2020;9:1244-51.

9. Zeng J, Lai H, Zheng D, et al. Effective treatment of ketamine-associated cystitis with botulinum toxin type a injection combined with bladder hydrodistention. J Int Med Res 2017;45:792-7.

10. Lee YK, Jhang JF, Kuo HC. Clinical Outcome of Augmentation Enterocystoplasty for Patients with Ketamine-induced Cystitis. Pain Physician 2017;20:E431-6.

11. Ng CF, Chiu PK, Li ML, et al. Clinical outcomes of augmentation cystoplasty in patients suffering from ketamine-related bladder contractures. Int Urol Nephrol 2013;45:1245-51.

12. Bao JM, Tan WL, Wang BW, et al. Transurethral frontfiring Greenlight bladder autoaugmentation for bladder contracture: technique and clinical outcomes. Int Urol Nephrol 2016;48:475-80.

13. Hanno PM, Erickson D, Moldwin R, et al. Diagnosis and treatment of interstitial cystitis/bladder pain syndrome: AUA guideline amendment. J Urol 2015;193:1545-53.

14. Morgan CJ, Rees H, Curran HV. Attentional bias to incentive stimuli in frequent ketamine users. Psychol Med 2008;38:1331-40.

15. Jhang JF, Birder LA, Chancellor MB, et al. Patient

Cite this article as: Tan S, Zhu X, Zheng Z, Zheng L, Kang Y, Tang Z. Comparison of bladder autoaugmentation by transurethral vesicomyotomy and hydrodistention for ketamine cystitis. Transl Androl Urol 2021;10(6):2351-2361. doi: 10.21037/ tau-21-188 characteristics for different therapeutic strategies in the management ketamine cystitis. Neurourol Urodyn 2017;36:687-91.

16. Johnson HW, Nigro MK, Stothers L, et al. Laboratory variables of bladder autoaugmentation in an animal model. Urology 1994;44:260-3.

17. Rocha FT, Bruschini H, Figueiredo JA, et al. Use of an inflatable silicone balloon improves the success rate of bladder autoaugmentation at long-term followup. J Urol 2011;185:2576-81.

18. Yee CH, Lai PT, Lee WM, et al. Clinical Outcome of a Prospective Case Series of Patients With Ketamine Cystitis Who Underwent Standardized Treatment Protocol. Urology 2015;86:236-43.

19. Dunn M, Ramsden PD, Roberts JB, et al. Interstitial cystitis, treated by prolonged bladder distension. Br J Urol 1977;49:641-5.

20. Cartwright PC, Snow BW. Bladder autoaugmentation: early clinical experience. J Urol 1989;142:505-8; discussion 520-1.

21. Cartwright PC, Snow BW. Bladder autoaugmentation: partial detrusor excision to augment the bladder without use of bowel. J Urol 1989;142:1050-3.

22. Stothers L, Johnson H, Arnold W, et al. Bladder autoaugmentation by vesicomyotomy in the pediatric neurogenic bladder. Urology 1994;44:110-3.

23. Wu P, Wang Q, Huang Z, et al. Clinical staging of ketamine-associated urinary dysfunction: a strategy for assessment and treatment. World J Urol 2016;34:1329-36.

24. Glemain P, Rivière C, Lenormand L, et al. Prolonged hydrodistention of the bladder for symptomatic treatment of interstitial cystitis: efficacy at 6 months and 1 year. Eur Urol 2002;41:79-84. 